\title{
Erratum: Bypass rewiring and robustness of complex networks [Phys. Rev. E 94, 022310 (2016)]
}

\author{
Junsang Park and Sang Geun Hahn \\ (Received 25 September 2017; published 25 October 2017)
}

DOI: 10.1103/PhysRevE.96.049904

The technical errors corrected here do not affect the results since the analytical derivations and the simulations in the previously published paper originally were based on the corrected descriptions and equations.

The word "error" in Sec. I should be corrected to "errors."

$p_{k} \sim k^{3}$ in the caption of Fig. 3 should be corrected to $p_{k} \sim k^{-3}$.

The expression in Sec. III, " $q_{k}$ is the probability that a randomly chosen link reaches a node with degree $k$," should be corrected to " $q_{k}$ is the probability that a randomly chosen link reaches a node with degree $k+1 . "$

The expression in Sec. III, " $u$ is the average probability that a randomly chosen node is not connected to the giant component," should be corrected to " $u$ is the average probability that a randomly chosen link is not connected to the giant component."

$\left(\phi=\phi_{k}\right)$ in Sec. III should be corrected to $\left(\phi_{k}=\phi\right)$.

The expression "The even degree network for Fig. 3(b) is generated" in Sec. III should be corrected to "The even degree network for Fig. 3(b) is generated randomly."

The sentence "At $t$-th step for $1 \leqslant t \leqslant\left\lfloor\frac{k}{2}\right\rfloor$, choose $\alpha^{\prime}$ which satisfies $R_{\alpha^{\prime}}=0$ and $Q_{\alpha^{\prime}} \geqslant Q_{i}$ for all $i$." should be corrected to "At the $t$ th step for $1 \leqslant t \leqslant\left\lfloor\frac{k}{2}\right\rfloor$, choose $\alpha^{\prime}$ which satisfies $R_{\alpha^{\prime}}=0$ and $Q_{\alpha^{\prime}} \geqslant Q_{i}$ with $R_{i}=0$ for all $i$."

The sentence "From chosen $\alpha^{\prime}$, choose $\alpha$ which satisfies $R_{\alpha}=0, Q_{\alpha}=Q_{\alpha^{\prime}}$, and $S_{\alpha} \geqslant S_{i}$ for all $i$." should be corrected to "From the chosen $\alpha^{\prime}$, choose the $\alpha$ which satisfies $R_{\alpha}=0, Q_{\alpha}=Q_{\alpha^{\prime}}$, and $S_{\alpha} \geqslant S_{i}$ with $R_{i}=0$ and $Q_{i}=Q_{\alpha^{\prime}}$ for all $i$."

The sentence "Otherwise, choose $\beta^{\prime}$ which satisfies $R_{\beta^{\prime}}=0, T_{\alpha, \beta^{\prime}}=0, Q_{\beta^{\prime}} \geqslant Q_{i}$ for all $i$." should be corrected to "Otherwise, choose $\beta^{\prime}$ which satisfies $R_{\beta^{\prime}}=0, T_{\alpha, \beta^{\prime}}=0, Q_{\beta^{\prime}} \geqslant Q_{i}$ with $R_{i}=0$ and $T_{\alpha, i}=0$ for all $i . "$

The sentence "From chosen $\beta^{\prime}$, choose $\beta$ which satisfies $R_{\beta}=0, T_{\alpha, \beta}=0, Q_{\beta}=Q_{\beta^{\prime}}$, and $S_{\beta} \geqslant S_{i}$ for all $i$." should be corrected to "From the chosen $\beta^{\prime}$, choose the $\beta$ which satisfies $R_{\beta}=0, T_{\alpha, \beta}=0, Q_{\beta}=Q_{\beta^{\prime}}$, and $S_{\beta} \geqslant S_{i}$ with $R_{i}=0, T_{\alpha, i}=0$, and $Q_{i}=Q_{\beta^{\prime}}$ for all $i$."

Equation $S_{\alpha}=S_{\beta}=S_{\alpha}+S_{\beta}$ in Sec. IV should be corrected to $S_{i}=\left[1-\left(1-T_{\alpha, i}\right)\left(1-T_{\beta, i}\right)\right]\left(S_{\alpha}+S_{\beta}\right)$ for all $i$.

The sentences in Sec. IV, "Update $T_{\alpha, i}=T_{i, \alpha}=1$ if there exists $i$ which satisfies $T_{\beta, i}=T_{i, \beta}=1$. Update $T_{\beta, i}=T_{i, \beta}=1$ if there exists $i$ which satisfies $T_{\alpha, i}=T_{i, \alpha}=1$." should be corrected to "Update $T_{i, j}=T_{j, i}=1$ if there exist $i$ and $j$ which satisfy $T_{i, \alpha} T_{\beta, j}=1$, that is, $T_{i, j}=T_{j, i}=1-\left(1-T_{i, j}\right)\left(1-T_{i, \alpha} T_{\beta, j}\right)$."

In Eq. (15), $Q_{i, j}$ should be corrected to $Q_{i}$.

$N(1-\phi)$ and $S$ in the labels of Fig. 4 should be deleted.

Reference [10] S. Boccaletti, V. Latora, M. Marchiori, and A. Rapisarda, Phys. Rep. 424, 175 (2006) should be corrected to [10] S. Boccaletti, V. Latora, Y. Moreno, M. Chavez, and D.-U. Hwang, Phys. Rep. 424, 175 (2006).

Reference [16] S. N. Dorogovtsev and A. V. Goltsev, Rev. Mod. Phys. 80, 1275 (2008) should be corrected to [16] S. N. Dorogovtsev, A. V. Goltsev, and J. F. F. Mendes, Rev. Mod. Phys. 80, 1275 (2008). 